\title{
Oviposición de Capnodis tenebrionis L. (Col. : Buprestidae) en ambiente controlado
}

\author{
José MALAGÓN, Antonio GARRIDO \& Teresa DEL BUSTO \\ I.V.I.A., Departamento de Protección Vegetal, Apartado Oficial, Moncada, Valencia, España
}

RESUMEN

SUMMARY
Se ha conseguido que $C$. tenebrionis realice la oviposición en ambiente controlado sin necesidad de recibir insolación directa. La experiencia se relizó en una cámara climatizada con temperatura constante de $25 \pm 1{ }^{\circ} \mathrm{C}$ y H.R. del $60 \pm 5$ p. 100 . Adicionalmente, las jaulas de puesta recibian luz infrarroja para elevar la temperatura hasta $30 \pm 2{ }^{\circ} \mathrm{C}$ durante 9 horas diarias y luz fluorescente ( $1400-1500$ lux) durante 15 horas.

En un ensayo realizado en la época natural de puesta se recogieron más huevos en ambiente controlado que en condiciones climáticas naturales, la oviposición se distribuyó más regularmente y se prolongó el período de puesta. También se recogieron huevos en un ensayo realizado fuera de la época natural de puesta (nov.-dic.), aunque en menor cantidad que en el ensayo anterior.

Palabras clave adicionales : Albaricoquero, frutales. conditions.

Oviposition of $C$. tenebrionis to was achieved under controlled laboratory conditions and without direct sun exposure. A chamber with constant temperature, $25 \pm 1{ }^{\circ} \mathrm{C}$ and $60 \pm 5 \% \mathrm{R}$. H. was used. Additionally, the egg-laying cages received infrared light to increase temperature up to $30 \pm 2{ }^{\circ} \mathrm{C}$ for $9 \mathrm{~h}$ daily and fluorescent light (1 400-1 500 lux) for $15 \mathrm{~h}$.

In a trial conducted during the natural egg-laying season, more eggs were obtained under controlled laboratory conditions ; moreover, the egg-laying being more uniform, there was an extension of the oviposition period. In a trial conducted out of the natural egg-laying season (Nov.-Dec.), some eggs were collected, but fewer than in the previous experiment.

Additional key words : Apricot, fruit trees.

Ponte de Capnodis tenebrionis $L$. (Col. Buprestidae) sous ambiance contrôlée.

Il a été acquis que $C$. tenebrionis pondait sous ambiance contrôlée, sans être exposé à une insolation directe. L'expérience a été réalisée dans une chambre climatisée (température de $25 \pm 1^{\circ} \mathrm{C}$, hygrométrie relative de $60 \pm 5 \mathrm{p} .100$ ). Les cages de ponte recevaient une lumière infrarouge additionnelle pour y élever la température jusqu'à $30 \pm 2{ }^{\circ} \mathrm{C}$ pendant $9 \mathrm{~h}$ et lumière fluorescente $(1400-1500$ lux $)$ pendant $15 \mathrm{~h}$ par jour.

Dans un essai effectué à l'époque naturelle de la ponte, plus d'œufs ont été déposés sous ambiance contrôlée que sous les conditions climatiques naturelles, la ponte étant plus régulière et prolongée. En dehors de l'époque naturelle de la ponte (novembre-décembre), des œeufs ont aussi été déposés, bien qu'en moins grand nombre que dans l'essai précédent.

Mots clés additionnels : Abricotier, fruitiers. 


\section{INTRODUCCION}

Capnodis tenebrionis $\mathbf{L}$. es un coleóptero de la familia Buprestidae perjudicial para los árboles frutales de hueso. Los daños causados en los árboles frutales de pepita son escasos y sin trascendencia económica.

Según BALACHOWSKY (1962), el área de distribución de $C$. tenebrionis comprende los paises ribereños del Mediterráneo y de las regiones circundantes, si bien su presencia ha sido señalada en varias regiones de Europa Central.

En España, ASCÁRATE (1893) lo cita por primera vez en el siglo pasado como perjudicial en los árboles de hueso y pepita en el término municipal de Jérez (Cádiz). Posteriormente ha causado daños en las regiones limítrofes con el Mediterráneo y en otras zonas frutícolas del interior peninsular (DOMíNGUEZ, 1944, 1976 ; Del CAÑIZO, 1950-51). Recientemente se han realizado estudios sobre la bioecología de este bupréstido en la región de Valencia (GARRIDO, 1984 ; GARRIDO \& DEL BUSTO, 1986) y en Andalucia (CABEZUELO et al., 1986).

Los daños causados por $C$. tenebrionis se han incrementado considerablemente en los últimos años, como consecuencia de la prolongada sequía habida en las zonas frutícolas españolas. En la región de Valencia, las plantaciones de albaricoquero y cerezo tienen a esta plaga como factor limitante por las pérdidas que ha causado en los últimos años.

Los estudios que se están realizado sobre la bioecología y control de los estados inmaduros de este insecto se encuentran con la dificultad de que el período de tiempo en el que se pueden conseguir huevos es relativemente corto, dado que en el medio natural, comprende desde finales de mayo hasta primeros de septiembre en la región de Valencia (GARRIDO, 1984).

El presente trabajo tiene por objeto conseguir alargar el período de oviposición, poniendo a los insectos adultos en condiciones climáticas similares a las del medio natural durante la época de puesta.

\section{MATERIAL Y METODOS}

\section{A. Características de la instalación}

La experiencia se realizó en una cámara climatizada, con una temperatura constante de $25 \pm 1{ }^{\circ} \mathrm{C}$ y $60 \pm 5$ p. $100 \mathrm{HR}$, en la que se instalaron compartimentos para ubicar las jaulas de puesta (fig. 1). Las jaulas recibian un complemento de uz y calor, para simular las condiciones ambientales del período de oviposición, mediante tubos fluorescentes que proporcionaban una iluminación de 1400 a 1500 lux en el interior de las jaulas durante $15 \mathrm{~h}$ al dia y lamparas infrarrojas que aseguraban una temperatura en el interior de las jaulas de $30 \pm 2{ }^{\circ} \mathrm{C}$ durante $9 \mathrm{~h}$ al dia, temperatura adecuada para la realización de la puesta (GAIRAUD) \& BESSON, 1950 ; KAITAZOV, 1958 ; BALACHOWSKY, 1962 ; MOURIKIS \& VASILAINA-AlEXOPOULOU, 1975). Para la recogida de huevos se siguió el método descrito por GARRIDO et al. (1987), realizándose los conteos cada 5 días.

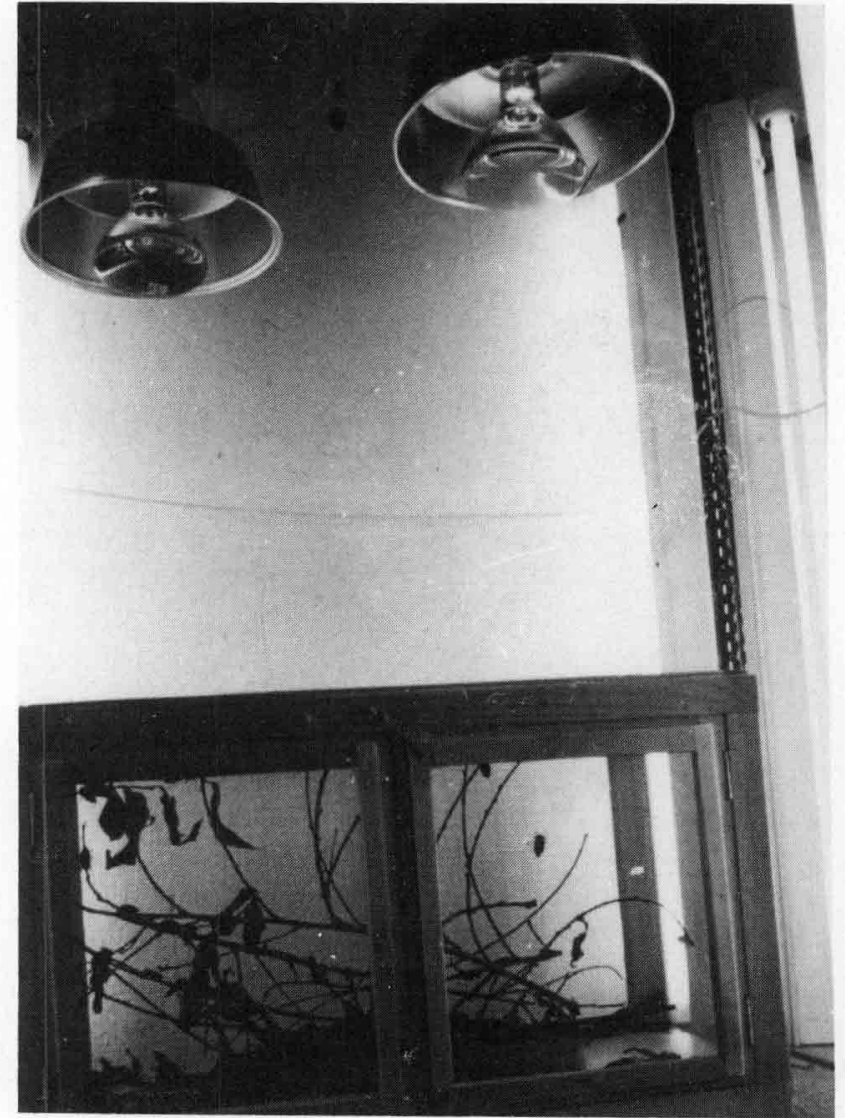

Figura 1

Jaulas de puesta ubicadas en el compartimento de la cámara climatizada.

Egg-laying cages placed in a compartment of the growth chamber. Cages de ponte placées dans un compartiment de la chambre climatisée.

\section{B. Material biológico}

Los ensayos se realizaron con insectos adultos recogidos en campo, en la zona del valle de Albaida, en los meses de agosto y octubre. Durante la experiencia los insectos se alimentaron con ramos de melocotonero de 40-60 cm de longitud que se renovaba cada 3-4 días. La experiencia terminó al morir todos los insectos del ensayo correspondiente.

\section{Desarrollo de la experiencia}

La experiencia constaba de dos ensayos :

\section{Obtención de huevos en la época natural}

Los insectos utilizados en este ensayo se recogieron a principios de agosto, época de máxima puesta (GARRIDO, 1984). Se formaron dos grupos de 12 parejas cada uno que se metieron en ambas jaulas de puesta. Una de las jaulas se puso en el exterior, en condiciones climáticas naturales y la otra en uno de los compartimentos de la cámara preparados al respecto.

\section{Obtención de huevos fuera de la época natural}

Los insectos utilizados en este ensayo se recogieron a mediados de octubre. En esta época no hay puesta en 
condiciones naturales en la zona del valle de Albaida (GARRIDO, 1984). Se formaron dos grupos de 32 parejas cada uno y se procedió de la forma descrita anteriormente.

\section{RESULTADOS}

Los resultados de estos ensayos se encuentran en la tabla 1. Puede observarse que tanto en el exterior como en ambiente controlado hubo continuidad en la puesta en la época natural, aún estando los insectos fuera de su medio natural, y que la puesta fue mayor en ambiente controlado. Fuera de la época natural de puesta sólo ovipositaron los insectos que estaban en ambiente controlado, aunque el número de huevos recogidos fue menor que en la época natural ; estos insectos estuvieron alimentándose durante un mes antes del comienzo de la puesta.
En la figura 2 se muestra una gráfica de la puesta en un periodo de la época natural. Se observa que la puesta fue más uniforme y el período de oviposición se prolongó en un ambiente controlado. En condiciones naturales la puesta aumentó al subir la temperatura máxima hasta $30-31{ }^{\circ} \mathrm{C}$ para disminuir posteriormente aunque la temperatura continuó aumentando. Después la temperatura máxima descendió por debajo de los $26{ }^{\circ} \mathrm{C}$, hacia finales de agosto, lo que se correspondió con un minimo en la puesta. Finalmente hubo un aumento de la puesta al aumentar la temperatura.

\section{DISCUSION Y CONCLUSION}

En la época natural de puesta, los resultados obtenidos sobre la influencia de la temperatura en la oviposición (fig. 2) coinciden con los hallados en anteriores investigaciones (FÉRON, 1949 ; CHRESTIAN, 1955;

TABLA 1

Puesta de C. tenebrionis en condiciones naturales y en ambiente controlado en dos periodos : en la época natural de puesta y fuera de ella. Oviposition of C. tenebrionis under field conditions and under controlled laboratory conditions in 2 periods : in/out of natural egg-laying season. Ponte de C. tenebrionis en conditions naturelles et dans une ambiance contrôlée de laboratoire : durant et après l'époque naturelle de ponte.

\begin{tabular}{|c|c|c|c|c|}
\hline \multirow{2}{*}{\multicolumn{2}{|c|}{ Fecha y control }} & \multicolumn{3}{|c|}{ Número de huevos recogidos } \\
\hline & & \multirow{2}{*}{$\begin{array}{c}\text { En el exterior (a) } \\
\begin{array}{c}\text { Insectos recogidos } \\
\text { en agosto }\end{array}\end{array}$} & \multicolumn{2}{|c|}{ En condiciones controladas } \\
\hline Mes-1986 & Dia & & $\begin{array}{c}\text { Insectos recogidos } \\
\text { en agosto }\end{array}$ & $\begin{array}{c}\text { Insectos recogidos } \\
\text { en octubre }\end{array}$ \\
\hline \multirow[t]{5}{*}{ Agosto : } & 8 & 0 & 0 & \\
\hline & 13 & 243 & 106 & \\
\hline & 18 & 760 & 165 & \\
\hline & 23 & 214 & 290 & \\
\hline & 28 & 162 & 364 & \\
\hline \multirow[t]{6}{*}{ Septiembre : } & 2 & 8 & 362 & \\
\hline & 7 & 205 & 284 & \\
\hline & 12 & 47 & 114 & \\
\hline & 17 & 15 & 60 & \\
\hline & 22 & 0 & 39 & \\
\hline & 27 & & 0 & \\
\hline \multirow[t]{6}{*}{ Octubre : } & 2 & & 16 & \\
\hline & 7 & & 0 & \\
\hline & 12 & & 0 & \\
\hline & 17 & & 6 & 0 \\
\hline & 22 & & 0 & 0 \\
\hline & 27 & & & 0 \\
\hline \multirow[t]{6}{*}{ Noviembre: } & 1 & & & 0 \\
\hline & 6 & & & 0 \\
\hline & 11 & & & 0 \\
\hline & 16 & & & 3 \\
\hline & 21 & & & 48 \\
\hline & 26 & & & 130 \\
\hline \multirow[t]{7}{*}{ Diciembre : } & 2 & & & 140 \\
\hline & 7 & & & 265 \\
\hline & 12 & & & 165 \\
\hline & 17 & & & 190 \\
\hline & 22 & & & 24 \\
\hline & 27 & & & 0 \\
\hline & TOTAL : & 1654 & 1826 & 965 \\
\hline
\end{tabular}

(a) Los insectos recogidos en octubre no realizarón puesta.

The insects collected in October did not lay eggs.

Les insectes collectés en octobre ne réaliseront pas de ponte. 


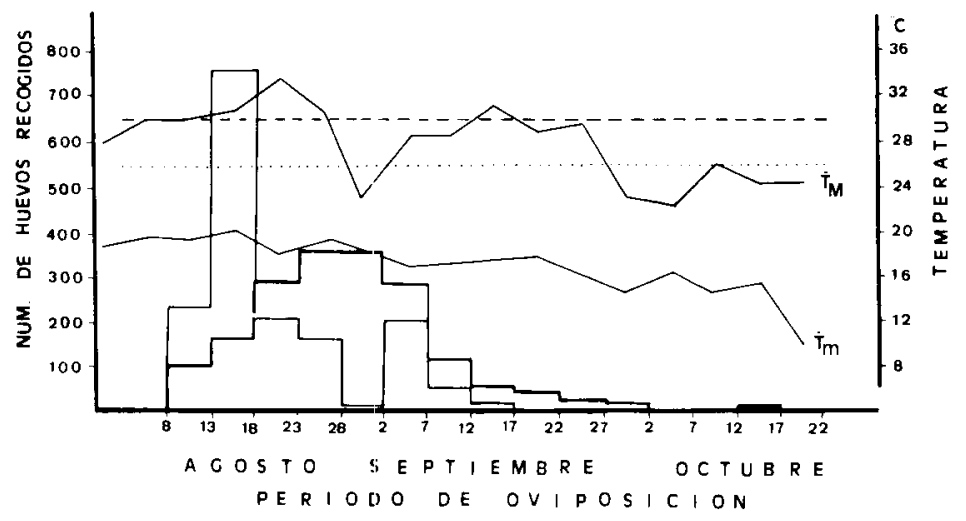

Figura 2

Gráficas de la oviposición de C. tenebrionis en condiciones naturales $y$ en ambiente controlado en un periodo de la época natural de puesta en 1986.

Oviposition graphs of $\mathrm{C}$. tenebrionis under field conditions and under controlled laboratory conditions in a period of naturai egglaying season in 1986.

Courbes de l'oviposition de $\mathrm{C}$. tenebrionis en conditions naturelles et dans une ambiance contrôlée de laboratoire dans une période de l'époque naturelle de ponte en 1986.

- Oviposición en condiciones naturales.

Oviposition under field conditions.

Oviposition en conditions naturelles.

- Oviposición en ambiente controlado.

Oviposition under controlled laboratory conditions.

Oviposition sous une ambiance contrôlée.

-_. - Temperatura media en las jaulas de puesta en ambiente controlado.

Mean temperature in the egg-laying cages under controlled laboratory conditions.

Température moyenne dans les cages de ponte sous ambiance contrôlée.

REICHART, 1967). La mayor oscilación de la temperatura en el exterior y la posible reabsorción de los óvulos antes de la ovulación por el descenso de la temperatura mínima (RICHARDS \& DAVIES, 1983) hacía el final de la puesta, pueden ser las causas de la reducción de la puesta en condiciones naturales respecto a la obtenida en condiciones controladas (tabla. 1).

Fuera de la época natural de puesta, hacia el final del otoño, la oviposición es menor que en verano (tabla. 1). Esto puede explicarse por la dificultad de suministrar a los insectos una alimentación adecuada ; comparable a los ramos que proporcionan en verano los árboles frutales y que es condición necesaría para que $C$. tenebrionis alcance la maduración sexual (GAIRAUD \& BESSON, 1950 : DEL CAÑIZO, 1950-51 ; MARTIN, 1951 ; ALAVIDZE, 1965 ; REICHART, 1967).
Temperatura media diaria a partir de la cual se produce la puesta (FERON, 1949).

Daily mean temperature for the oviposition to begin (FERON, 1949).

Température journalière moyenne à partir de laquelle se produit la ponte (FERON, 1949).

$\bar{T}_{M} \quad$ Temperatura media de las máximas en condiciones naturales. Mean upper temperatures under field conditions.

Températures movennes maximales en conditions naturelles.

$\bar{T}_{m} \quad$ Temperatura media de las mínimas en condiciones naturales. Mean lower temperatures under field conditions.

Températures movennes minimales en conditions naturelles.
Así pués, es posible reproducir en condiciones controladas las necesidades ambientales requeridas por $C$. tenebrionis en cuanto a la temperatura $(9 \mathrm{~h}$ diarias a $30^{\circ} \mathrm{C}$ ) y luminosidad ( $15 \mathrm{~h}$ diarias con $1500 \mathrm{lux}$ ) para realizar la puesta, ampliando así el período en que es posible disponer de huevos para profundizar las investigaciones sobre los estados inmaduros de este insecto.

Reçu le 5 mai 1987. Accepté le 19 janvier 1988.

\section{AGRADECIMIENTOS}

Los autores desean expresar su agradecimiento a Magdalena VIL CHES por la mecanografía del trabajo y a Ana Borrás por la traducción al inglés y francés del resumen y leyendas de las gráficas y figuras.

REFERENCIAS BIBLIOGRAFIAS

Alavidze B. A., 1965. Some supplementary data on the biology of the black buprestid (Capnodis tenebrionis L.) in Georgia and measures for its control. Zashch. Rast., 17, 37-48 (en russe).

Ascárate C., 1893. Insectos y Criptogamas que invaden los cultivos en España. Tipolitografía de L. Péant e Hijos, Madrid, $780 \mathrm{p}$.
Balachowsky A. S., 1962. Entomologie Appliquée d̀ l'Agriculture. Coléoptères. 1 vol., Masson et Cie. Editeurs, Paris, 564 p.

Cabezuelo P., Varona M. J., Rivas N., Soriano M. L., Fernández M., Fernández F. J., 1986. Contribución al conocimiento de la biología del « gusano cabezudo » (Capnodis tenebrionis L..) en Andalu- 
cia. $2^{e}$ Symposium Nacional de Agroquímicos, Junta de Andalucia, Consellería de Agricultura y Pesca, Sevilla, 57-69.

Chrestian P., 1955. Le Capnode noir des Rosacées. Protectorat de la République Française au Maroc, Service de la Défense des Végétaux, Travaux originaux $\mathrm{n}^{\circ} 6$, Rabat (Maroc), $141 \mathrm{p}$.

Del Cañizo J., 1950-51. Una plaga de los frutales de hueso : El "gusano cabezudo" (Capnodis tenebrionis L.). Bol. Patol. Veg. Entomol. Agric., 13, 429-446.

Domínguez F., 1944. Las plagas de los frutales en Espana y su distribución geográfica. II. Coleópteros y Dípteros. Bol. Patol. Veg. Entomol. Agric., 13, 429-446.

Domínguez F., 1976. Plagas y Enfermedades de las plantas cultivadas. Editorial Dossat, S.A., Madrid, $5^{e}$ ed., 955 p.

Féron M., 1949. Recherches sur la ponte de Capnodis tenebrionis L. (Col., Buprestidae). Rev. Pathol. vég., 28, 66-72.

Gairaud R., Besson J., 1950. Contribution à l'étude de la biologie du burpreste du pêcher (Capnodis tenebrionis L.) dans la Mitidja (Algérie). Rev. Pathol. vég., 29, 119-136.

Garrido A., 1984. Bioecología de Capnodis tenebrionis L. (Col., Buprestidae) y orientaciones para su control. Bol. Serv. Plagas., 10, 205-221.
Garrido A., Del Busto T., 1986. El gusano cabezudo (Capnodis tenebrionis L. ; Col. : Buprestidae). Agricola Vergel., 49, 23-29.

Garrido A., Del Busto T., Malagón J., 1987. Método de recogida de huevos de Capnodis tenebrionis L. (Col. : Buprestidae) y algunos factores abióticos que pueden condicionar la puesta. Bol. San. Veg. Plagas., 13, 303-309.

Kaitazov A., 1958. Capnodis tenebrionis L. Bionomics and measure for control. Zasht. Rast., 1, 159-187. (En búlgaro).

Martin H., 1951. Contribution à l'étude du capnode noir des arbres fruitiers (Capnodis tenebrionis L.) dans la région d'Alger. Rev. Pathol, vég., 30, 97-113.

Mourikis P. A., Vasilaina-Alexopolou P., 1975. Uber die Laborzucht und Entwicklung des Pfirsich-Prachtkäfers, Capnodis tenebrionis L. (Col., Buprestidae). Anz. Schädlingskde, Pflanzenschutz, Umweltschutz., 48, 75-77.

Reichart G., 1967. New data to the biology of Capnodis tenebrionis L. (Coleoptera). Acta Zool. hung., 13, 395-408.

Richards O. W., Davies R. G., 1983. Tratado de Entomología Imms. 1 vol., Editorial Omega, S.A., Barcelona, 438 p. 